\title{
Epidemiology of pharyngeal carriage of Fusobacterium necrophorum
}

Fusobacterium necrophorum is the cause of Lemierre's disease, a life-threatening septicaemia secondary to a septic thrombophlebitis of the internal jugular vein preceded by acute sore throat (Hagelskjær et al., 1998). Despite its importance as an invasive pathogen, uncertainty surrounds its carriage, significance as a primary cause of sore throat and its mode of acquisition. We have sought to shed light on these issues in a study involving two different groups: university students and patients presenting to local general practitioners (GPs) with a sore throat.

In December 2005 and January 2006, throat swabs were taken from 411 university students (range 18-39 years, median 20 years, males $40.6 \%$, females $59.1 \%$, unknown sex $0.2 \%$ ) and 103 patients who presented to local GPs with sore throat (range 2-77 years, median 26 years, males $60 \%$, females $39 \%$, unknown sex $1 \%)$. Throat swabs were tested for $\beta$ haemolytic streptococci by routine culture and Epstein-Barr virus (EBV) DNA and $F$. necrophorum by real-time PCR, employing laboratory methods described previously (Patel et al., 2003; Aliyu et al., 2004). In addition, the students completed a questionnaire for possible risk factors for throat infection. Chi-square tests (employing Yates' correction for two by two contingency tables) and Fisher's exact tests using StatsDirect software were used to determine whether there were significant differences in frequencies of epidemiological factors and other differences between the F. necrophorum PCR-positive and -negative groups. Odds ratios were calculated using the Woolf (logit) approximation and conditional likelihood estimation was used to calculate exact Fisher two-sided $P$-values. Ethical

A supplementary table giving full results for the analysis of risk factors associated with a positive Fusobacterium necrophorum test is available with the online version of this paper. approval for the study, which included informed consent, was granted by the Local Research Ethics Committee.

Overall, 43/411 (10.5\%) students were positive for $F$. necrophorum and this represented asymptomatic carriage in 29/ $43(67.4 \%)$ of these. Students who tested positive for $\beta$-haemolytic streptococci and/ or EBV were excluded from the analysis for epidemiological risk factors as it was considered that the presence of these infections could be a potential confounder. In addition, a student with a clinical diagnosis of streptococcal sore throat who had recently received intravenous antibiotic treatment was excluded. Implementing these exclusion criteria left 362 students remaining for the risk factor analysis.

Analysis of the possible risk factors (excluding those students with $\beta$ haemolytic streptococcal infection and/or EBV infection) associated with a positive test showed no significant differences for age, sex, subject studied, presence of sore throat currently or in the previous 2 weeks, two or more episodes of sore throat over the previous 6 months, current smoking status, sharing a bedroom, sharing a house, contact with a person with a sore throat in the last 4 weeks, contact with cats, dogs or other animals on at least a weekly basis, and antibiotic treatment currently or in the preceding 4 weeks. However, those who were F. necrophorum-positive were significantly more likely to have had one or more lip-to-lip kissing contacts in the previous 4 weeks $(26 / 30,86.7 \%)$ than those who were negative $(212 / 317,66.9 \%)$ [Odds ratio (OR) 3.22 (1.10-9.46), $P=0.04]$. See Supplementary Table S1 in JMM Online for full results.

For both student (including those excluded from the risk factor analysis) and GP patient groups separately, we found that those with $F$. necrophorum were significantly more likely to carry EBV or a $\beta$-haemolytic streptococcus, or both, than those who were negative [students $11 / 43$ as compared with 38/368, OR 2.99 (1.396.40); GP patients $10 / 20$ as compared with 20/83, OR 3.15 (1.15-8.65)].

EBV infection is acquired from humans by intimate oral contact with others and shares with Lemierre's disease a peak incidence of infection in late adolescence (Niederman \& Evans, 1997), and our findings suggest the source and route of transmission to be the same for $F$. necrophorum. Tully et al. (2006) have reported that adolescent students are at increased risk of meningococcal disease due to frequent intimate oral contact with multiple partners. The increased transmission of throat pathogens in this social group doubtless accounts for our finding of co-infection of F. necrophorum with EBV and $\beta$-haemolytic streptococci and may also explain the increased likelihood of asymptomatic carriage of $F$. necrophorum in this social group compared with the healthy non-student adults we have studied previously (Aliyu et al., 2004); 29 of the 411 students $(7.1 \%)$ were asymptomatic carriers compared with 0 of $100(P=0.01)$ in the previously studied healthy non-student adults.

It has been suggested that F. necrophorum is not a primary pathogen in the throat, invasive disease being facilitated by prior infection with a putative higher grade pathogen, in particular EBV, as coinfection has been reported in case studies of Lemierre's disease (Dagan \& Powell, 1987; Alvarez \& Schreiber, 1995). Evidence for a primary role for $F$. necrophorum as a cause for sore throat would be provided by finding a positive association between having a sore throat and a positive $F$. necrophorum test, particularly if those carrying other throat pathogens are excluded from the analysis. Excluding students with EBV or $\beta$-haemolytic streptococci, there was a higher proportion of students who were positive for $F$. necrophorum who had had a sore throat in 
the previous 2 weeks $(10 / 32,31.3 \%)$ than the proportion who had had a sore throat in the F. necrophorum-negative group (75 of $330,22.7 \%$ ), but this did not achieve statistical significance [OR 1.55 (0.703.41)]. However, we were underpowered to prove this association as, following exclusions, comparatively few students remained in the study who reported a sore throat ( 85 of $362,23.5 \%$ ). The evidence for a possible important primary role of $F$. necrophorum in patients with acute sore throat has been supported by a recent report that F. necrophorum was second only to group A streptococci as a bacterial pathogen in this setting (Amess et al., 2007). Notably, the isolation rate was highest (12.9\% of samples) in 16-20 year olds in this study. Further case-control studies will be required to prove a causal role in acute sore throat, taking into account the level of carriage in adolescents. Batty et al. (2005) have suggested an important role in recurrent sore throat syndrome, but this was not confirmed in our study; students reported the occurrence of two or more episodes of sore throat in the preceding 6 months no more commonly when carrying the organism $(15 / 27,55.5 \%)$ than when negative (176/317, 55.5\%) [OR 1.00 (0.45-2.21, $P>0.99)]$. However, our study may have been underpowered to prove the association and this also requires further investigation.

Whilst our previous study (Aliyu et al., 2004) implied that finding F. necrophorum in the throat swab of a non-student indicated clinical infection, the same was not true for this predominantly adolescent student group, where the recovery of $F$. necrophorum did not necessarily indicate clinical infection (although some may have developed a sore throat later). However, we found that the magnitude of the copy count per swab of F. necrophorum may give an indication of the likelihood of active clinical infection; when we combined the F. necrophorum-positive students and
F. necrophorum-positive GP patients we found that 7 out of $20(35 \%)$ subjects with a sore throat and without EBV or $\beta$ haemolytic streptococci had copy counts of 50000 or more, whereas this finding only applied to 1 of $22(4.6 \%)$ asymptomatic subjects $(P=0.03$, Fisher's exact test). Therefore, the presence of a swab copy count of 50000 or more in a symptomatic individual suggests that $F$. necrophorum is the cause of the sore throat. This concurs with the findings of Jensen et al. (2007), who reported that in their study of 18-32 year olds comparing patients with non-streptococcal tonsillitis and asymptomatic healthy controls, the load of F. necrophorum was significantly higher in the symptomatic individuals with nonstreptococcal tonsillitis.

In conclusion, although asymptomatic carriage appears to be rare in healthy older subjects, this appears common in the predominantly adolescent student population group in this study. Our findings indicate that throat carriage of $F$. necrophorum in university students is related to social lifestyle, being transmitted with other throat pathogens by intimate human mouth-to-mouth contact. Clinical infection may be distinguished from asymptomatic carriage by a swab copy count above 50000 .

\section{Hugo Ludlam, ${ }^{1}$ Julia Howard, ${ }^{1,2}$ Barry Kingston, ${ }^{3}$ Linda Donachie, ${ }^{1}$ Joanne Foulkes, ${ }^{1}$ Simantee Guha ${ }^{1}$ and Martin D. Curran ${ }^{1}$}

${ }^{1}$ Clinical Microbiology and Public Health Laboratory, Addenbrooke's Hospital, Cambridge CB2 2OW, UK

${ }^{2}$ East of England Public Health Training Programme, East of England MultiProfessional Deanery, Ida Darwin Site, Fulbourn, Cambridge CB21 5EE, UK

${ }^{3}$ University of Cambridge Department of Pathology, Tennis Court Road, Cambridge CB2 1OP, UK
Correspondence: Hugo Ludlam (hugo.ludlam@addenbrookes.nhs.uk)

Aliyu, S. H., Marriott, R. K., Curran, M. D., Parmar, S., Bentley, N., Brown, N. M., Brazier, J. S. \& Ludlam, H. (2004). Real-time PCR investigation into the importance of Fusobacterium necrophorum as a cause of acute pharyngitis in general practice. $J$ Med Microbiol 53, 1029-1037.

Alvarez, A. \& Schreiber, J. R. (1995). Lemierre's syndrome in adolescent children - anaerobic sepsis with internal jugular vein thrombophlebitis following pharyngitis. Pediatrics 96, 354-359.

Amess, J. A., O'Neill, W., Giollariabhaigh, C. N. \& Dvtrych, J. K. (2007). A six-month audit of Fusobacterium necrophorum from patients with sore throat in a district general hospital. $\mathrm{Br} \mathrm{J}$ Biomed Sci 64, 63-65.

Batty, A., Wren, M. W. \& Gal, M. (2005). Fusobacterium necrophorum as the cause of recurrent sore throat: comparison of isolates from persistent sore throat syndrome and Lemierre's disease. J Infect 51, 299-306.

Dagan, R. \& Powell, K. R. (1987). Postanginal sepsis following infectious mononucleosis. Arch Intern Med 147, 1581-1583.

Hagelskjær, L. H., Prag, J., Malczynski, J. \& Kristenen, J. H. (1998). Incidence and clinical epidemiology of necrobacillosis, including Lemierre's syndrome, in Denmark 1990-1995. Eur J Clin Microbiol Infect Dis 17, 561-565.

Jensen, A., Hagelskjaer Kristensen, L. \& Prag, J. (2007). Detection of Fusobacterium necrophorum subsp. funduliforme in tonsillitis in young adults by real-time PCR. Clin Microbiol Infect 13, 695-701.

Niederman, J. C. \& Evans, A. S. (1997). EpsteinBarr virus. In Viral Infections of Humans: Epidemiology and Control, pp. 253-283. Edited by A. S. Evans \& R. A. Kaslow. New York \& London: Plenum Medical Book Company.

Patel, S., Zuckerman, M. \& Smith, M. (2003). Real-time quantitative PCR of Epstein-Barr virus BZLF1 DNA using the LightCycler. J Virol Methods 109, 227-233.

Tully, J., Viner, R. M., Coen, P. G., Stuart, J. M., Zambon, M., Peckham, C., Booth, C., Klein, N., Kaczmarski, E. \& Booy, R. (2006). Risk and protective risk factors for meningococcal disease in adolescents: matched cohort study. BMJ 332, 445-450. 